\title{
A General Approach to Convert Hemicyanine Dyes into Highly Optimized Photoacoustic Scaffolds for Analyte Sensing
}

\author{
Sarah H Gardner ${ }^{\dagger[a]}$, Catharine J Brady ${ }^{\dagger[b]}$, Cameron Keeton ${ }^{[b]}$, Anuj K Yadav ${ }^{[b]}$, Sharath C Mallojjala[c], \\ Melissa Y Lucero ${ }^{[b]}$, Shengzhang Su ${ }^{[b]}$, Zhengxin $\mathrm{Yu}^{[b]}$, Jennifer S Hirschi[c], Liviu M Mirica ${ }^{[b]}$, Jefferson \\ Chan*[a,b]
}

† These authors contributed equally

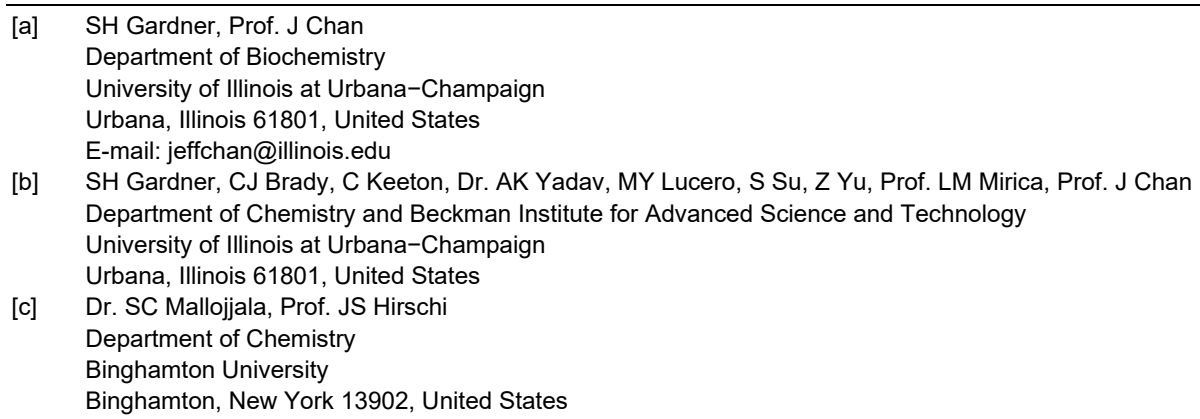

Supporting information for this article is given via a link at the end of the document.

\begin{abstract}
In the context of deep-tissue disease biomarker detection and analyte sensing of biologically relevant species, the impact of photoacoustic imaging has been profound. However, most photoacoustic imaging agents to date are based on the repurposing of existing fluorescent dye platforms that exhibit non-optimal properties for photoacoustic applications (e.g., high fluorescence quantum yield). Herein, we introduce two effective modifications to the hemicyanine dye to afford PA-HD, a new dye scaffold optimized for photoacoustic probe development. We observed a significant increase in the photoacoustic output, representing an increase in sensitivity of 4.8-fold and a red-shift of the $\lambda_{\text {abs }}$ from $690 \mathrm{~nm}$ to $745 \mathrm{~nm}$ to enable ratiometric imaging. Moreover, to demonstrate the generalizability and utility of our remodeling efforts, we developed three probes using common analyte-responsive triggers for betagalactosidase activity (PA-HD-Gal), nitroreductase activity (PA-HDNTR), and hydrogen peroxide (PA-HD- $\left.\mathrm{H}_{2} \mathrm{O}_{2}\right)$. The performance of each probe (responsiveness, selectivity) was evaluated in vitro and in cellulo. To showcase the enhance properties afforded by PA-HD for in vivo photoacoustic imaging, we employed an Alzheimer's disease model to detect $\mathrm{H}_{2} \mathrm{O}_{2}$. In particular, the photoacoustic signal at 735 $\mathrm{nm}$ in the brains of 5xFAD mice (a murine model of Alzheimer's disease) increased by $1.79 \pm 0.20$-fold relative to background indicating the presence of oxidative stress, whereas the change in wildtype mice was negligible $(1.02 \pm 0.14)$. These results were confirmed via ratiometric calibration which was not possible using the parent HD platform.
\end{abstract}

\section{Introduction}

Photoacoustic imaging is a powerful in vivo approach that is characterized by the generation of ultrasound waves which result from the excitation of an optical absorber with light. ${ }^{1,2}$ In theory, any light-absorbing material can be induced to afford a photoacoustic readout; however, in practice, only molecules with a wavelength of maximum absorbance in the near-infrared (NIR) region $\left(\lambda_{\text {abs }}>650 \mathrm{~nm}\right)$, a high extinction coefficient $\left(\epsilon>10^{4} \mathrm{M}^{-1} \mathrm{~cm}\right.$ $\left.{ }^{1}\right)$ and a low quantum yield $(\Phi<5 \%)$ are desirable. Initially, when photoacoustic imaging emerged as an in vivo biomedical technique, various endogenous absorbers such as hemoglobin, oxyhemoglobin, and melanin were exploited to provide contrast. These studies set the stage for label-free imaging of various cancer types and inflammatory conditions in human patients. ${ }^{3}$ However, as the demand for improved performance, better contrast, greater target specificity, and molecular information grew, efforts turned toward the design of targeted contrast agents ${ }^{4}$ and activatable photoacoustic probes (also known as acoustogenic probes) $)^{5,6}$ to reliably identify diseased tissue and to monitor biological analytes, respectively.

Owing to the intrinsic similarities between photoacoustic and fluorescence imaging (i.e., the requirement of light excitation), it is reasonable that most photoacoustic imaging agents to date have relied heavily on the repurposing of existing fluorophores. For example, indocyanine green (ICG), an FDA-approved cyanine-based fluorescent dye, was utilized extensively in early studies for contrast-enhanced photoacoustic imaging even though it is prone to photobleaching and oxidative decomposition. Likewise, the first small-molecule activatable photoacoustic probe (designed to detect $\left.\mathrm{Cu}^{2+}\right)^{7}$ was based on the aza-BODIPY dye, which is another NIR fluorescent platform. ${ }^{8}$ Subsequent studies have resulted in a palette of aza-BODIPY-based photoacoustic probes for hypoxia, ${ }^{9-11}$ nitric oxide, ${ }^{12-14}$ peroxynitrite, ${ }^{15}$ hydrogen peroxide, ${ }^{16} \mathrm{pH},{ }^{17}$ redox status, ${ }^{18}$ and photodynamic therapy. ${ }^{19,} 20$ In addition, our group put forth significant efforts to optimize this scaffold for photoacoustic imaging; however, this required the handling of unstable species such as reactive azirine intermediates, as well as challenging (and sometimes low yielding) purifications to separate the desired heteropyrrole coupling product from the unwanted symmetrical aza-BODIPYs. ${ }^{21}$ 
Due to these limitations, our design has not yet been widely adopted by other probe developers. Beyond aza-BODIPYs, other NIR fluorescent platforms such as cyanines ${ }^{22-30}$ and hemicyanine dyes (HD) $)^{31-39}$ have also been repurposed extensively (Scheme 1a).

HDs have attracted considerable attention from the field owing to their ease of synthesis (one-step starting from a parent cyanine and resorcinol) and the presence of a convenient handle (i.e., $\mathrm{OH}$ or $-\mathrm{NH}_{2}$ ) from which analyte-responsive triggers can be appended to. ${ }^{40}$ Unfortunately, HDs are far from ideal for photoacoustic analyte sensing. First, the $\lambda_{\text {abs }}$ of a 'capped' probe $(\sim 60)$ typically falls below the NIR cutoff of $650 \mathrm{~nm}$, as well as the lower wavelength limit of our commercial photoacoustic imaging systems (660 nm (MSOT iNVision, iThera) and $680 \mathrm{~nm}$ (Nexus 128+, Endra)). These constraints preclude ratiometric calibration which can account for imaging artifacts that result from photobleaching or differential probe clearance. Second, HDs are highly fluorescent molecules ( $\Phi=\sim 30-40 \%$ ), meaning less of the excited state will relax via non-radiative decay to afford a photoacoustic signal. In this study, we introduce two highly effective modifications to transform existing HD designs into optimized scaffolds for photoacoustic imaging by substituting the endocyclic oxygen with a sulfur moiety and by tuning the phenolic pKa value (herein referred to as PA-HDs). To demonstrate generalizability, we prepared three enhanced photoacoustic probes from PA-HD for $\beta$-galactosidase activity, nitroreductase activity, and hydrogen peroxide $\left(\mathrm{H}_{2} \mathrm{O}_{2}\right)$. Beyond cellular studies in which all three probes were tested, we also employed our $\mathrm{H}_{2} \mathrm{O}_{2}$ probe to image oxidative stress in a murine model of Alzheimer's disease.

\section{Results and Discussion}

The design of PA-HD is based on the premise that sulfursubstitution can potentially red-shift the $\lambda_{\text {abs }}$ value by up to $50 \mathrm{~nm}$ and thus, can facilitate deeper tissue imaging and ratiometric calibration. Moreover, we anticipate two additional benefits from this modification that favor photoacoustic imaging. First, we hypothesized the fluorescent quantum yield will be attenuated and second, we expected the extinction coefficient to be enhanced. It is noteworthy that although the apparent pKa of regular HDs (herein referred to as O-HD) was reported to be $\sim 5.6,{ }^{40}$ this value appears to be inaccurate. In our hands we measured a value of 7.5 (Figure S1). This discrepancy likely resulted because the $\mathrm{pH}$ of each buffer system used to construct the corresponding $\mathrm{pH}$-profile was not adjusted after the organic co-solvent component was added. A high pKa value will decrease the photoacoustic sensitivity and lead to imaging artifacts since the protonated form of O-HD will have a similar absorbance to that of the unactivated probe. Therefore, in addition to performing the proposed $\mathrm{O}$ to $\mathrm{S}$ substitution, it is critical to install an ortho chloro group to lower the phenolic pKa which ensures that upon unmasking of the trigger, the turned over PA-HD will exist predominantly in a deprotonated form. To this end, we synthesized PA-HD by first substituting the meso-chloro group of Cy7-Cl with thiophenol 1 to furnish cyanine 2 in $52 \%$ yield. This intermediate was then demethylated with $\mathrm{BBr}_{3}$ and heated under basic conditions after solvent exchange to initiate the retroKnoevenagel reaction to generate PA-HD in $64 \%$ yield over 2steps $(33 \%$ overall yield).
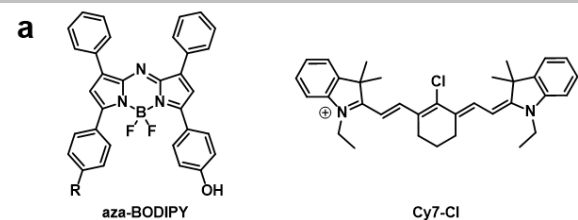

Cy7-Cl

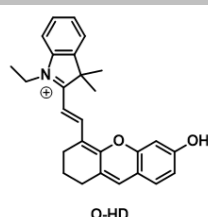

O.HD

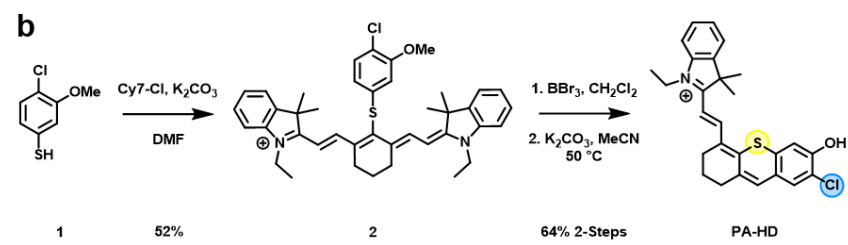

Scheme 1. a) Examples of NIR fluorescent dyes that have been repurposed for photoacoustic applications. b) Synthesis of PA-HD.

With PA-HD in hand, we first determined that the $\lambda_{\text {abs }}$ was positioned at $745 \mathrm{~nm}$ which represents a bathochromic shift of 55 $\mathrm{nm}$ relative to O-HD (Figure 1a). Moreover, it is apparent that at $\mathrm{pH} 7.4$, over half the population of O-HD is protonated and this results in a blue-shifted $\lambda_{\text {abs }}$ and low $\epsilon$. In contrast, since the apparent pKa value of PA-HD is 6.0 , over $96 \%$ of the dye will be deprotonated at physiological $\mathrm{pH}$ (Figure S2). We also found that the $\epsilon$ had increased significantly by $62 \%$ from $8.2 \times 10^{4} \mathrm{M}^{-1} \mathrm{~cm}^{-1}$ to $13.3 \times 10^{4} \mathrm{M}^{-1} \mathrm{~cm}^{-1}$ and that the $\Phi$ decreased by six-fold from $30 \%$ to $5 \%$. As mentioned previously, meeting these two design criteria will translate into enhanced photoacoustic properties. To evaluate
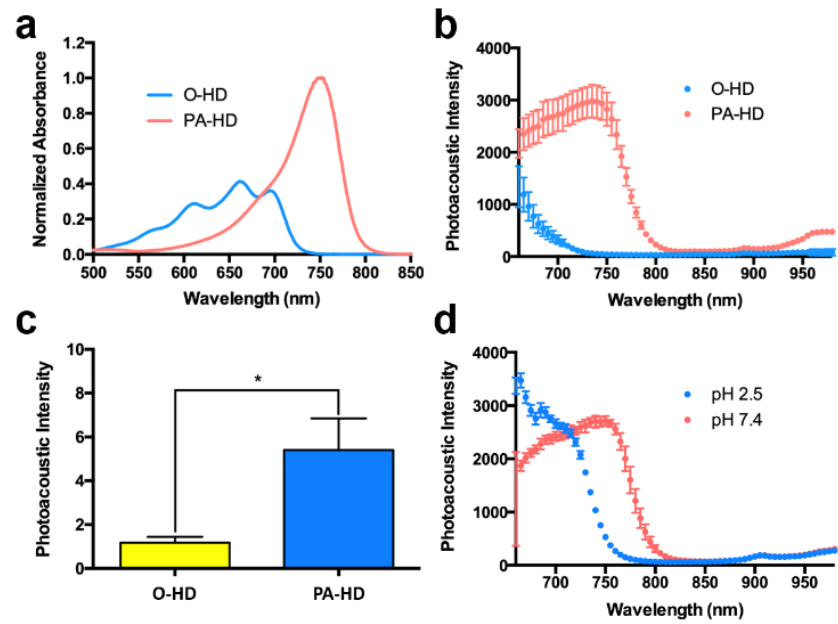

Figure 1. a) Absorbance and b) $\mathrm{PA}$ spectra of $\mathrm{O}-\mathrm{HD}$ and $\mathrm{PA}-\mathrm{HD}$ at $\mathrm{pH} 7.4$ (1:1 v/v PBS:EtOH). Dye concentration was $25 \mu \mathrm{M}$ for each dye. c) Normalized photoacoustic intensity of O-HD and PA-HD in a tissue phantom containing $60 \%$ milk to mimic scattering effects in dense tissue. $[\mathrm{PA}-\mathrm{HD}]=0.5 \mu \mathrm{M}(n=3)$. Statistical analysis was performed using twotailed Student's t-test $(\alpha=0.05),{ }^{*}: p<0.05$. d) Photoacoustic spectra of PA-HD at $\mathrm{pH} 2.5$ and 7.4 .

the impact of sulfur-substitution with respect to the photoacoustic signal intensity and potential ratiometric sensing capabilities, we conducted a series of head-to-head in vitro experiments. First, we embedded solutions of each dye $(25 \mu \mathrm{M})$ in an agar-based tissue phantom (10\% milk) and obtained photoacoustic spectra over a wavelength range of 660 to $980 \mathrm{~nm}$ (Figure 1b). While the O-HD $\lambda_{\text {abs }}$ clearly fell outside the NIR window, the highest intensity for PA-HD was centered at $\sim 735 \mathrm{~nm}$. This represents a 4.8 -fold increase in sensitivity when comparing the highest signals. 
Additionally, we show a dose-dependent increase in the photoacoustic intensity after correcting for wavelength-dependent differences in fluence (Figure S3). Next, we reduced the dye concentration to $0.5 \mu \mathrm{M}$ and changed the composition of milk in the phantom to $60 \%$ to mimic the scattering effects of dense tissue (e.g., brain). Even under these conditions that are designed to attenuate the photoacoustic signal, we could readily detect PAHD but not O-HD (Figure S4). The corresponding normalized photoacoustic intensities (relative to background) were $5.4 \pm 2.5$ fold and $1.18 \pm 0.47$-fold, respectively (Figure 1c). To evaluate the potential of this new scaffold for ratiometric imaging, we subjected PA-HD to acidic conditions since protonation of the phenol is an effective proxy for the presence of a trigger at this position. Indeed the protonated form of PA-HD exhibits a $\lambda_{\text {abs }}$ at $650 \mathrm{~nm}$ (Figure S5). The ratios of the deprotonated and protonated forms at 650 $\mathrm{nm}$ and $745 \mathrm{~nm}$ based on absorbance were found to be 0.36 and 16.81 , respectively (Figure 1d). This represents a theoretical turnon response of 46.7 (defined as ratio ${ }_{745} / \mathrm{ratio}_{650}$ ). Similar results were obtained when the experiment was performed at $660 \mathrm{~nm}$ (lower wavelength limit) and $735 \mathrm{~nm}$ (maximum signal) via photoacoustic imaging to give a turn-on response of 3.7. Together these results demonstrate PA-HD can be employed for ratiometric imaging, while this is not possible with O-HD.

Next, we converted PA-HD into a series of three activatable photoacoustic probes to demonstrate that the enhanced properties afforded by the sulfur-substitution are generalizable. Specifically, PA-HD-Gal (Figure 2a), PA-HD-NTR (Figure 2c), and PA-HD- $\mathrm{H}_{2} \mathrm{O}_{2}$ (Figure 2e) were developed for $\beta$-galactosidase activity, nitroreductase activity, and $\mathrm{H}_{2} \mathrm{O}_{2}$, respectively. We selected these three imaging targets because their corresponding triggers are commonly employed to validate new dye systems and they are important in a host of physiological and pathological processes. For instance, $\beta$-galactosidase expression has been used as a marker to identify senescent cells $^{41}$ and ovarian cancer. ${ }^{42}$ Nitroreductase has been used as a target to detect gram positive and negative bacteria, ${ }^{43}$ as well as tumor hypoxia since it is overexpressed in many cancer types. ${ }^{44}$ Lastly, $\mathrm{H}_{2} \mathrm{O}_{2}$ is not only an important signaling molecule, at elevated levels it is a general indicator of oxidative stress in a variety of disease states. ${ }^{45}$

After synthesizing the three probes, each was subjected to in vitro testing to assess target-responsiveness. We found that PA-HDGal was readily activated upon incubation with recombinant betagalactosidase. Specifically, a dose-dependent (0 to $10 \mathrm{U} / \mathrm{mL}$ ) change in the absorbance (shift of the $\lambda_{\text {abs }}$ from 640 to $745 \mathrm{~nm}$ ) was observed, representing a maximum signal enhancement of $9.1 \pm 0.5$-fold based on absorbance (Figure $2 \mathrm{~b}$ ). We confirmed that this increase was due to the enzyme target because heatinactivation of beta-galactosidase prior to incubation with PA-HDGal completely attenuated probe activation (Figure S6). Likewise, PA-HD-NTR was activated by recombinant nitroreductase from $E$. coli (19.8 \pm 1.7 -fold change in fluorescence after $1 \mathrm{~h})$. Moreover, the trigger was demonstrated to be stable in the presence of biological thiols such as $\mathrm{Cys}, \mathrm{H}_{2} \mathrm{~S}$, and $\mathrm{GSH}$ which can potentially reduce the nitro group and lead to 1,4-elimination to give a false response (Figure 2d). ${ }^{46}$ Finally, we determined that PA-HD- $\mathrm{H}_{2} \mathrm{O}_{2}$ retained its responsiveness to $\mathrm{H}_{2} \mathrm{O}_{2}$. Specifically, a $6.3 \pm 0.8$-fold and $11.9 \pm 1.5$-fold change in fluorescence was noted after incubation of the probe for $1 \mathrm{~h}$ with $100 \mu \mathrm{M}$ and $500 \mu \mathrm{M} \mathrm{H}_{2} \mathrm{O}_{2}$, respectively (Figure 2f). In contrast, when PA-HD- $\mathrm{H}_{2} \mathrm{O}_{2}$ was subjected to selectivity screening against a panel of reactive oxygen/nitrogen species (hypochlorite, nitric oxide, tert-butyl a

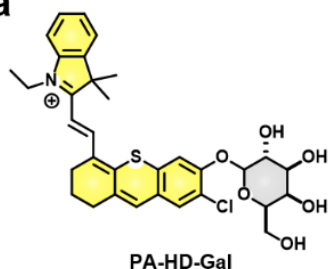

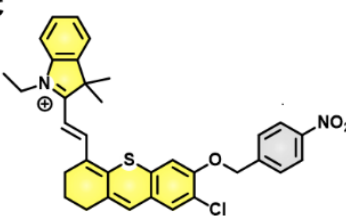

PA-HD-NTR b

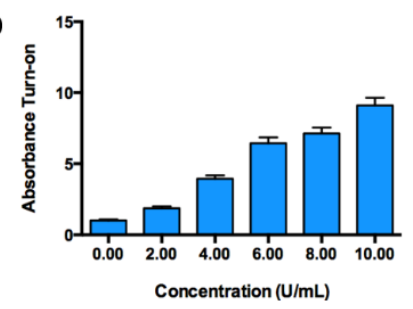

d

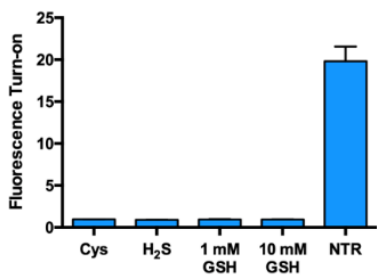

e
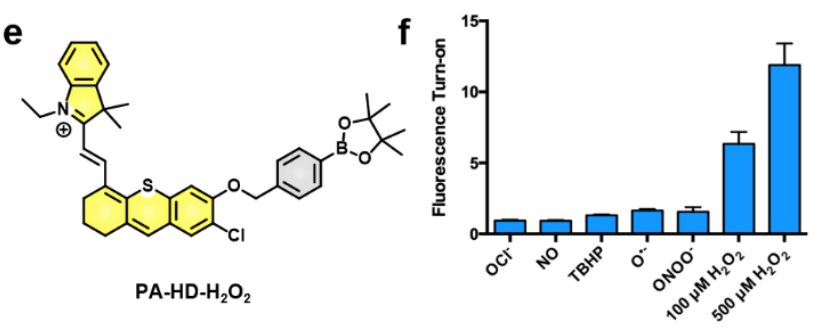

Figure 2. a) Chemical structure of PA-HD-Gal. b) Dose-dependent response of $5 \mu \mathrm{M}$ PA-HD-Gal after $1 \mathrm{~h}$ to $0,2,4,6,8$, or $10 \mathrm{U} / \mathrm{mL}$ of $E$. Coli beta-galactosidase at $\mathrm{pH} 7.4$ (9:1 v/v PBS:MeCN) (Mean $\pm \mathrm{SD}, n=5)$. c) Chemical structure of PA-HD-NTR. d) Response of $2 \mu \mathrm{M}$ PA-HD-NTR to biologically relevant thiols (Cys and $\mathrm{H}_{2} \mathrm{~S} 100 \mu \mathrm{M}$ ) and $\mathrm{GSH}$ at $1 \mathrm{mM}$ and $10 \mathrm{mM}$ for $15 \mathrm{~min}$ at $\mathrm{pH} 7.4$ (9:1 v/v PBS:MeCN) (Mean $\pm \mathrm{SD}, n=3$ ). Complete activation mediated by $2 \mathrm{U} / \mathrm{mL}$ E. coli nitroreductase. e) Chemical structure of PA-HD- $\mathrm{H}_{2} \mathrm{O}_{2}$. f) Response of $2 \mu \mathrm{M}$ PA-HD- $\mathrm{H}_{2} \mathrm{O}_{2}$ to $\mathrm{OCl}^{-}$, NO, TBHP, $\mathrm{O}_{2}^{-}$, ONOO- $(100 \mu \mathrm{M})$ and $\mathrm{H}_{2} \mathrm{O}_{2}(100 \mu \mathrm{M}$ and $500 \mu \mathrm{M})$ for $1 \mathrm{~h}$ at $\mathrm{pH} 7.4(9: 1 \mathrm{v} / \mathrm{v}$ PBS:MeCN) (Mean $\pm \mathrm{SD}, n=3)$.

hydroperoxide, superoxide, peroxynitrite) there was no significant turn-on. Collectively, these results show that installation of established triggers onto our new photoacoustic scaffold does not impact their performance. Additional in vitro characterization can be found in the supporting information document and the photophysical properties of all dyes are summarized below in Table 1.

Table 1. Summary of photophysical properties

\begin{tabular}{|c|c|c|c|c|c|c|}
\hline Compound & $\begin{array}{l}\lambda_{\mathrm{abs}}{ }^{\mathrm{a}} \\
(\mathrm{nm})\end{array}$ & $\begin{array}{l}\lambda_{\mathrm{em}}{ }^{\mathrm{a}} \\
(\mathrm{nm})\end{array}$ & $\begin{array}{c}\epsilon^{\mathrm{a}} \\
\left(10^{4} \mathrm{M}^{-1} \mathrm{~cm}^{-1}\right)\end{array}$ & $\begin{array}{c}\phi_{\mathrm{F}}{ }^{\mathrm{a}, \mathrm{b}} \\
(\%)\end{array}$ & $\begin{array}{c}\text { PABF } \\
\left(10^{4}\right)\end{array}$ & $\mathbf{p K a}^{\mathrm{c}}$ \\
\hline $\mathrm{O}-\mathrm{HD}$ & 690 & 712 & 8.2 & 30 & 5.7 & 7.5 \\
\hline PA-HD & 745 & 765 & 13.3 & 5 & 12.6 & 6.0 \\
\hline PA-HD-Gal & 640 & 728 & 3.8 & 0.7 & 3.8 & N.D. \\
\hline PA-HD-NTR & 642 & 735 & 2.7 & 0.7 & 2.8 & N.D. \\
\hline PA-HD- $\mathrm{H}_{2} \mathrm{O}_{2}$ & 645 & 738 & 2.8 & 0.7 & 2.7 & N.D. \\
\hline
\end{tabular}

a] Determined at $\mathrm{pH} 8.25$ in 1:1 v/v PBS:MeOH. [b] vs. ICG in DMSO. [c] pKa values determined in 1:1 v/v Britton-Robinson buffers ranging from $\mathrm{pH} 2.6$ to 11.3. N.D. = Not determinable. 


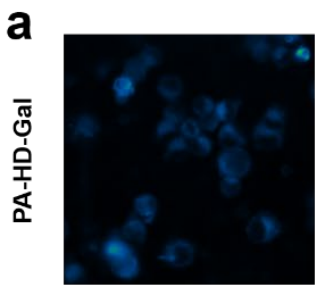

OVCAR-3

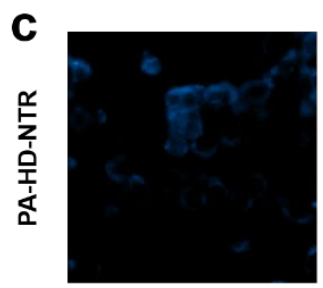

$20 \% \mathrm{O}_{2}$

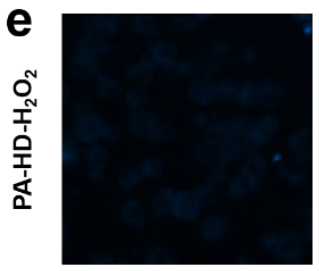

$0 \mu \mathrm{M} \mathrm{H}_{2} \mathrm{O}_{2}$

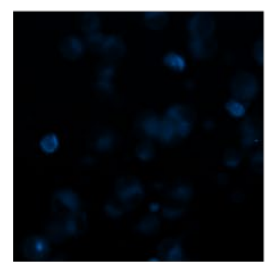

OVCAR-3 + BGA

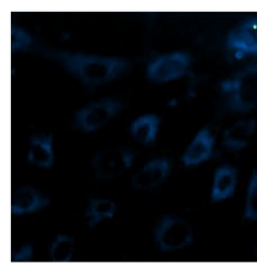

$5 \% \mathrm{O}_{2}$

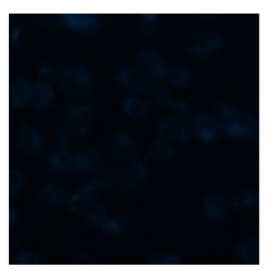

$25 \mu \mathrm{M} \mathrm{H}_{2} \mathrm{O}_{2}$

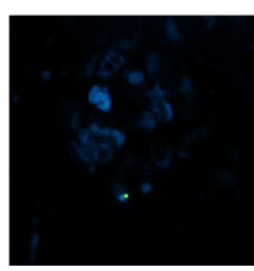

IGROV-1

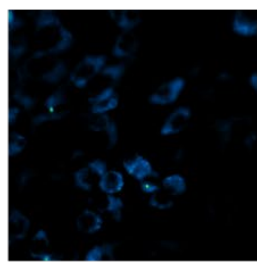

$2 \% \mathrm{O}_{2}$

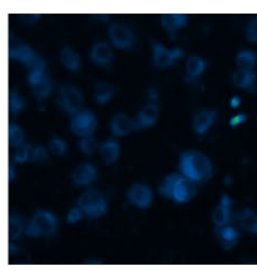

$50 \mu \mathrm{M} \mathrm{H}_{2} \mathrm{O}_{2}$

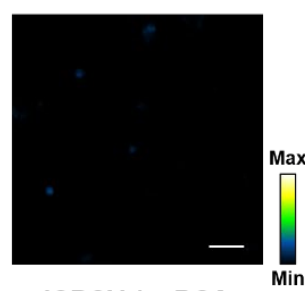

IGROV-1 + BGA

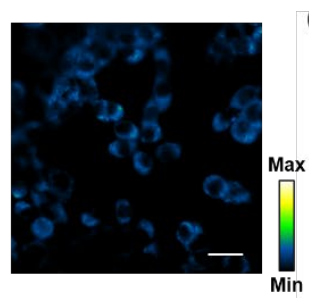

$1 \% \mathrm{O}_{2}$

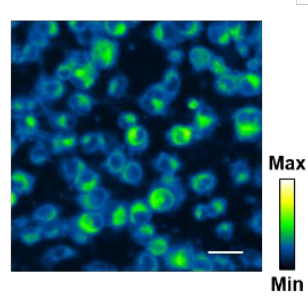

$100 \mu \mathrm{M} \mathrm{H}_{2} \mathrm{O}_{2}$ b

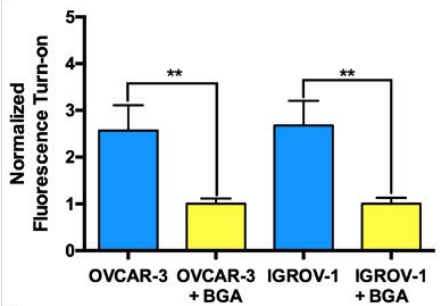

d

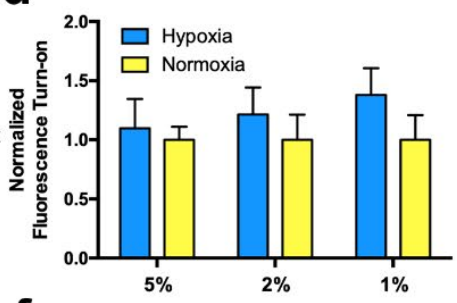

f

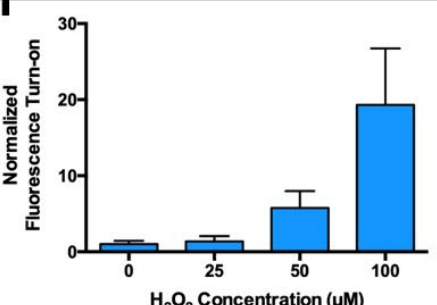

Figure 3. a) Fluorescent images of PA-HD-Gal (4 $\mu \mathrm{M})$ in ovarian cancer cell lines (OVCAR-3 and IGROV-1) pre-treated with a vehicle control or BGA, a betagalactosidase inhibitor (1.5mM). $n=3$ for each condition. Statistical analysis was performed using two-tailed Student's t-test $(\alpha=0.05)$, **: $p<0.01$. b) Quantified data from a) normalized to inhibitor condition. c) Fluorescent images of PA-HD-NTR (2 $\mu$ M) in ID8 cells cultured under the indicated oxygen atmosphere (20,5, 2, or $1 \%$ oxygen). $n=3$ for each condition. d) Quantified data from c) normalized to a normoxic control. e) Fluorescent Images of PA-HD$\mathrm{H}_{2} \mathrm{O}_{2}(10 \mu \mathrm{M})$ in NeuroScreen-1 cells treated with various concentrations of $\mathrm{H}_{2} \mathrm{O}_{2}(0,25,50100 \mu \mathrm{M})$. $n=3$ for each condition. f) Quantified data from e) normalized to the $0 \mu \mathrm{M} \mathrm{H}_{2} \mathrm{O}_{2}$ condition.

We utilized the MTT assay to evaluate cytotoxicity of PA-HD in several mammalian cell lines. Incubation of PA-HD for $6 \mathrm{~h}$ at concentrations up to $10 \mu \mathrm{M}$ did not significantly impact cell viability (Figure S7). With these results in hand, we focused our attention on evaluating the performance of each probe in cell-based studies. Beta-galactosidase is known to be expressed in ovarian cancer presumably because it is involved in the processing of glycans such as sialyl Lewis $x .{ }^{47}$ Upon incubation of two human ovarian cancer cell lines, OVCAR-3 and IGROV-1, with PA-HD-Gal we were able to observe an apparent cytosolic staining pattern which suggests our probe was being activated by beta-galactosidase (Figure 3a). To confirm these results, we prepared BGA, a potent beta-galactosidase inhibitor, ${ }^{48}$ to determine whether pretreatment would attenuate probe activation. Relative to OVCAR-3 and IGROV-1 cells that were not treated with the inhibitor, the signal intensity was 2.18-fold and 2.78-fold lower, respectively (Figure $3 b)$. Next, the ability of PA-HD-NTR to distinguish between normoxic and hypoxic conditions via nitroreductase activity was tested. 4T1 breast cancer cells were cultured in a $20 \%, 5 \%, 2 \%$, or $1 \%$ oxygen atmospheres for $\sim 12 \mathrm{~h}$ before treatment with PAHD-NTR. Under oxygen deficient conditions, nitroreductases present within cells can convert the aryl nitro group to the corresponding hydroxyl amine or amino moieties via multiple single electron transfer events. This reduction can facilitate unmasking of PA-HD via self-immolative chemistry. As anticipated, the signal was barely discernable from background when PA-HD-NTR was incubated under normoxic conditions $\left(20 \% \mathrm{O}_{2}\right)$ (Figure $3 \mathrm{c}$ ). In contrast, the intensity was $1.38 \pm 0.10$ fold higher at $1 \%$ oxygen (Figure $3 d$ ). Importantly, the low but statistically significant signal enhancement is a consequence of the trigger which requires chronic hypoxic conditions to upregulate nitroreductase expression. Finally, NeuroScreen-1 cells, a model system for neurons, was stained with PA-HD- $\mathrm{H}_{2} \mathrm{O}_{2}$ and treated with $0,25,50$, or $100 \mu \mathrm{M} \mathrm{H}_{2} \mathrm{O}_{2}$ (Figure $3 \mathrm{e}$ ). We selected this cell line because oxidative stress plays a central role in neurodegenerative diseases, as well as aging of the brain. Our results revealed a dose-dependent increase in the signal intensity where the highest concentration of $\mathrm{H}_{2} \mathrm{O}_{2}$ resulted in a $19.3 \pm 7.4$ fold turn-on response after incubation (Figure $3 \mathrm{f}$ ).

Finally, it is critical for us to evaluate whether the enhanced photoacoustic properties of PA-HD will effectively translate in a deep-tissue context. Owing to its excellent analyte selectivity profile and ability to detect $\mathrm{H}_{2} \mathrm{O}_{2}$ in a dose-dependent manner, we sought to employ PA-HD- $\mathrm{H}_{2} \mathrm{O}_{2}$ to image oxidative stress (via $\mathrm{H}_{2} \mathrm{O}_{2}$ detection) in an Alzheimer's disease model. The brain consumes massive amounts of oxygen to fuel its stringent metabolic demands. ${ }^{49}$ However, when the natural antioxidant defense systems of the brain are compromised, a net increase in the generation of reactive oxygen species will result. Since oxidative stress is a hallmark of many neurological disorders including Alzheimer's disease,,$^{50}$ it is reasonable to assume that $\mathrm{H}_{2} \mathrm{O}_{2}$ will be elevated in the brain; however, there is limited direct evidence showing this to be true at the molecular level. ${ }^{51,52}$ In our study, we systemically administered PA-HD- $\mathrm{H}_{2} \mathrm{O}_{2}$ to $5 x F A D$ mice which is a well-establish transgenic model engineered to express the human amyloid beta protein precursor. ${ }^{53}$ We employed photoacoustic imaging at $660 \mathrm{~nm}$ to track probe uptake and observed a signal 


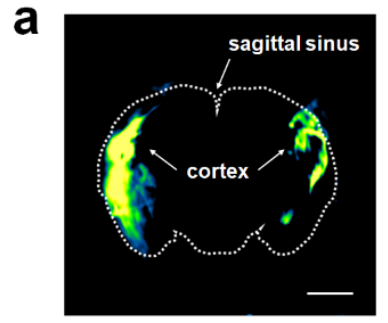

Alzheimer's Disease

C

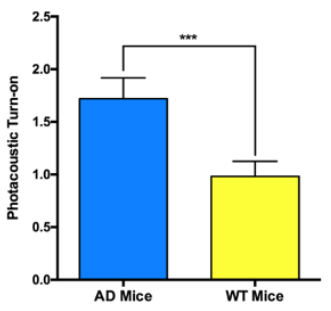

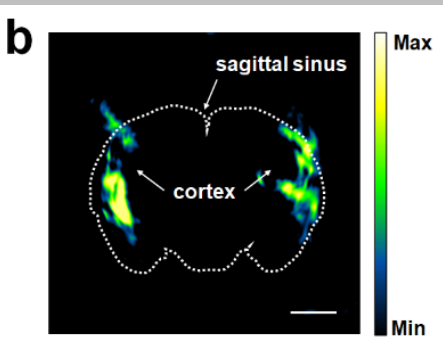

Wildtype

d

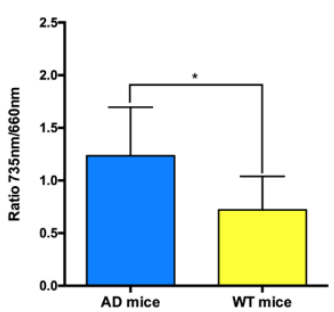

Figure 4. Representative photoacoustic images of the brain from a) an Alzheimer's disease mouse and b) a wildtype mouse captured $2.5 \mathrm{~h}$ after PA-HD- $\mathrm{H}_{2} \mathrm{O}_{2}$ was administered via retroorbital injection with excitation provided at $735 \mathrm{~nm}$. Scale bar represents $2.5 \mathrm{~mm}$. Dotted white outline of the brain is for visualization purposes only. Brain region cropped and overlayed onto a dark background. c) Photoacoustic turn-on response for Alzheimer's disease mice $(n=5)$ and wildtype mice $(n=6)$. Error bars = SD. Statistical analysis was performed using two-tailed Student's t-test ( $\alpha$ $=0.05),{ }^{* * *}: \mathrm{p}<0.001$. d) Photoacoustic ratio at $735 \mathrm{~nm} / 660 \mathrm{~nm}$ of data from c). Statistical analysis was performed using the Mann-Whitney $U$ test

increase at this wavelength that plateaued after $\sim 1 \mathrm{~h}$. To ensure sufficient time for probe activation, we waited a total of $2.5 \mathrm{~h}$ before irradiating the brain at $735 \mathrm{~nm}$ to detect the turned over product. A marked increase in the photoacoustic signal at this wavelength relative to the initial timepoint was observed $(1.79 \pm$ 0.20 -fold increase) indicating conversion of PA-HD- $\mathrm{H}_{2} \mathrm{O}_{2}$ to PA$\mathrm{HD}$. In contrast, the turn-on response for healthy wildtype controls (B6SJLF1/J mice) was only $1.02 \pm 0.14$-fold. To corroborate these results, we turned to ratiometric analysis. We hypothesized the $\mathrm{PA}_{735} / \mathrm{PA}_{660}$ ratio will be higher in the $5 \mathrm{xFAD}$ mice compared to the B6SJLF1/J mice at the $2.5 \mathrm{~h}$ timepoint since this would represent probe activation and generation of PA-HD. Indeed, the ratio for the $5 x F A D$ mice was $1.23 \pm 0.46$, whereas the ratio for the B6SJLF1/J mice was $0.85 \pm 0.07$. Ratiometric imaging would not have possible with O-HD.

\section{Conclusion}

At the onset of this study, our goal was to establish a new dye platform that is optimized for the development of activatable photoacoustic probes. This approach represents a significant departure from the common practice of repurposing NIR fluorescent dyes that exhibit sub-optimal properties for photoacoustic imaging. One of the key criteria that we prioritized when developing PA-HD was accessibility, since we believe a high synthetic overhead ( eight steps) would be less desirable to probe developers in the field. The O-HD dye developed by Lin and co-workers represents an attractive starting point for our remodelling efforts because it can be prepared from $\mathrm{Cy} 7-\mathrm{Cl}$ and resorcinol in a single step and has been shown to be a versatile platform for various biological applications. Beginning from 4chloro-3-methoxybenzenethiol, PA-HD can be prepared in only three-steps with an overall yield of $33.3 \%$. In this study, we demonstrated that substitution of the endocyclic oxygen atom with a sulfur group affords many desired properties. For instance, we observed a red-shifted $\lambda_{\text {abs }}$ of $55 \mathrm{~nm}$ which facilitated access to deeper tissue and enabled ratiometric imaging. The ability to confirm an in vivo imaging result by tracking the signal change at two wavelengths clearly sets PA-HD apart from O-HD. While spectral unmixing is another strategy we could have potentially utilized to isolate the signal of PA-HD- $\mathrm{H}_{2} \mathrm{O}_{2}$ from that of background, this requires us to obtain a high-quality in vivo spectrum of the probe which we were unable to do in this instance. This highlights why ratiometric imaging is so critical. It is noteworthy that while we were evaluating the photoacoustic properties of PA-HD, a group prepared a NIR fluorescent probe based on a similar platform (lacking the ortho-chloro group) to image cysteine in live cells. ${ }^{54}$ However, as we have demonstrated in this work, sulfur-substitution results in a significant decrease in the fluorescent quantum yield which favors photoacoustic imaging while rendering the dye less effective for optical imaging. Indeed, to acquire the fluorescent cell images featured in Figure 3, it was necessary for us to irradiate with the LED light source set at $100 \%$ power in some instances. With this in mind, we envision PA-HD will facilitate the development of a diverse range of new activatable photoacoustic probes. We are optimistic that PA-HD will become widely adopted by the photoacoustic field and this work will result in a divergence between O-HD-based probes for fluorescent applications and PA-HD-based chemical tools for photoacoustic imaging.

\section{Acknowledgements}

The authors acknowledge the National Institutes of Health for support (R35GM133581 to J.C.; R01GM114588 to L.M.M.). This work used the XSEDE computational resource which is supported by National Science Foundation grant number $\mathrm{ACl}-1548562$ allocation TG-CHE180061. C.J.B. acknowledges the ChemistryBiology Interface Training Grant (T32-GM136629) and Robert C. and Carolyn J. Springborn Graduate Fellowship for support. M.Y.L. thanks the Alfred P. Sloan Foundation for financial support. Major funding for the $500 \mathrm{MHz}$ Bruker CryoProbeTM was provided by the Roy J. Carver Charitable Trust (Muscatine, lowa; Grant No. 15-4521) to the School of Chemical Sciences NMR Lab. The Q-Tof Ultima mass spectrometer was purchased in part with a grant from the National Science Foundation, Division of Biological Infrastructure (DBI-0100085). We also thank Prof. Erik R. Nelson (Molecular and Integrative Physiology, UIUC) for ID8 and IGROV-1 cells and Prof. Kai Zhang (Biochemistry, UIUC) for NeuroScreen-1 cells. Furthermore, we thank Dr. Sandy McMasters (UIUC Cell Media Facility) for help with preparation of cell culture media and technical expertise and Dr. Andrew Brannen (iThera Medical) for helpful suggestions.

Keywords: Photoacoustic imaging • Acoustogenic Probe • Analyte Sensing $\bullet$ Hydrogen Peroxide $•$ Alzheimer's Disease 
[1] Wang, L. V., and Hu, S. (2012) Photoacoustic Tomography: In Vivo Imaging from Organelles to Organs, Science 335, 1458.

[2] Yadav, A. K., Hernandez, S., Su, S., and Chan, J. (2020) Acoustic-based chemical tools for profiling the tumor microenvironment, Current Opinion in Chemical Biology 57, 114-121.

[3] Steinberg, I., Huland, D. M., Vermesh, O., Frostig, H. E., Tummers, W. S., and Gambhir, S. S. (2019) Photoacoustic clinical imaging, Photoacoustics 14, 77-98.

[4] Weber, J., Beard, P. C., and Bohndiek, S. E. (2016) Contrast agents for molecular photoacoustic imaging, Nature Methods 13, 639-650.

[5] Reinhardt, C. J., and Chan, J. (2018) Development of Photoacoustic Probes for in Vivo Molecular Imaging, Biochemistry 57, 194-199.

[6] Knox, H. J., and Chan, J. (2018) Acoustogenic Probes: A New Frontier in Photoacoustic Imaging, Accounts of Chemical Research 51, 2897-2905.

[7] Li, H., Zhang, P., Smaga, L. P., Hoffman, R. A., and Chan, J. (2015) Photoacoustic Probes for Ratiometric Imaging of Copper(II), Journal of the American Chemical Society 137, 15628-15631.

[8] Ge, Y., and O'Shea, D. F. (2016) Azadipyrromethenes: from traditional dye chemistry to leading edge applications, Chemical Society Reviews 45, 3846-3864.

[9] Knox, H. J., Hedhli, J., Kim, T. W., Khalili, K., Dobrucki, L. W., and Chan, J. (2017) A bioreducible N-oxide-based probe for photoacoustic imaging of hypoxia, Nature Communications 8, 1794

[10] Knox, H. J., Kim, T. W., Zhu, Z., and Chan, J. (2018) Photophysical Tuning of $\mathrm{N}$-Oxide-Based Probes Enables Ratiometric Photoacoustic Imaging of Tumor Hypoxia, ACS Chemical Biology 13, 1838-1843.

[11] Chen, M., Knox, H. J., Tang, Y., Liu, W., Nie, L., Chan, J., and Yao, J. (2019) Simultaneous photoacoustic imaging of intravascular and tissue oxygenation, Opt. Lett. 44, 37733776.

[12] Reinhardt, C. J., Zhou, E. Y., Jorgensen, M. D., Partipilo, G., and Chan, J. (2018) A Ratiometric Acoustogenic Probe for in Vivo Imaging of Endogenous Nitric Oxide, Journal of the American Chemical Society 140, 1011-1018.

[13] Zhou, E. Y., Knox, H. J., Reinhardt, C. J., Partipilo, G., Nilges, M. J., and Chan, J. (2018) Near-Infrared Photoactivatable Nitric Oxide Donors with Integrated Photoacoustic Monitoring, Journal of the American Chemical Society 140, 11686-11697.

[14] Reinhardt, C. J., Xu, R., and Chan, J. (2020) Nitric oxide imaging in cancer enabled by steric relaxation of a photoacoustic probe platform, Chemical Science 11, 15871592.

[15] Zhang, J., Zhen, X., Upputuri, P. K., Pramanik, M., Chen, P., and Pu, K. (2017) Activatable Photoacoustic Nanoprobes for In Vivo Ratiometric Imaging of Peroxynitrite, Advanced Materials 29, 1604764.

[16] Lu, X., Zhao, M., Chen, P., Fan, Q., Wang, W., and Huang, W. (2018) Enhancing hydrophilicity of photoacoustic probes for effective ratiometric imaging of hydrogen peroxide, Journal of Materials Chemistry B 6, 4531-4538.

[17] Geng, J., Li, W., Smaga, L. P., Sottos, N. R., and Chan, J. (2018) Damage-Responsive Microcapsules for Amplified Photoacoustic Detection of Microcracks in Polymers, Chemistry of Materials 30, 2198-2202.

[18] Zheng, J., Zeng, Q., Zhang, R., Xing, D., and Zhang, T. (2019) Dynamic-Reversible Photoacoustic Probe for Continuous Ratiometric Sensing and Imaging of Redox Status in Vivo, Journal of the American Chemical Society 141, 1922619230.

[19] Tang, Q., Si, W., Huang, C., Ding, K., Huang, W., Chen, P., Zhang, Q., and Dong, X. (2017) An aza-BODIPY photosensitizer for photoacoustic and photothermal imaging guided dual modal cancer phototherapy, Journal of Materials Chemistry B 5, 1566-1573.

[20] Gawale, Y., Adarsh, N., Kalva, S. K., Joseph, J., Pramanik, M., Ramaiah, D., and Sekar, N. (2017) Carbazole-Linked Near-Infrared Aza-BODIPY Dyes as Triplet Sensitizers and Photoacoustic Contrast Agents for Deep-Tissue Imaging, Chemistry - A European Journal 23, 6570-6578.

[21] Zhou, E. Y., Knox, H. J., Liu, C., Zhao, W., and Chan, J. (2019) A Conformationally Restricted Aza-BODIPY Platform for Stimulus-Responsive Probes with Enhanced Photoacoustic Properties, Journal of the American Chemical Society 141, 17601-17609.

[22] Mishra, A., Jiang, Y., Roberts, S., Ntziachristos, V., and Westmeyer, G. G. (2016) Near-Infrared Photoacoustic Imaging Probe Responsive to Calcium, Analytical Chemistry 88, 10785-10789.

[23] Zhang, J., Smaga, L. P., Satyavolu, N. S. R., Chan, J., and Lu, Y. (2017) DNA Aptamer-Based Activatable Probes for Photoacoustic Imaging in Living Mice, Journal of the American Chemical Society 139, 17225-17228.

[24] Roberts, S., Seeger, M., Jiang, Y., Mishra, A., Sigmund, F., Stelzl, A., Lauri, A., Symvoulidis, P., Rolbieski, H., Preller, M., Deán-Ben, X. L., Razansky, D., Orschmann, T., Desbordes, S. C., Vetschera, P., Bach, T., Ntziachristos, V., and Westmeyer, G. G. (2018) Calcium Sensor for Photoacoustic Imaging, Journal of the American Chemical Society 140, 2718-2721.

[25] Xiao, H., Wu, C., Li, P., Gao, W., Zhang, W., Zhang, W., Tong, L., and Tang, B. (2017) Ratiometric photoacoustic imaging of endoplasmic reticulum polarity in injured liver tissues of diabetic mice, Chemical Science 8, 7025-7030.

[26] Liu, Y., Wang, S., Ma, Y., Lin, J., Wang, H.-Y., Gu, Y., Chen, X. and Huang, P. (2017) Ratiometric Photoacoustic Molecular Imaging for Methylmercury Detection in Living Subjects, Advanced Materials 29, 1606129.

[27] Ma, G., Gao, X., Jiang, C., Xing, S., Wei, C., Huang, P., and Lin, J. (2019) pH-Responsive Nanoprobe for In Vivo Photoacoustic Imaging of Gastric Acid, Analytical Chemistry 91, 13570-13575.

[28] Zhang, Y., He, S., Chen, W., Liu, Y., Zhang, X., Miao, Q., and Pu, K. (2021) Activatable Polymeric Nanoprobe for NearInfrared Fluorescence and Photoacoustic Imaging of $T$ Lymphocytes, Angewandte Chemie International Edition 60, 5921-5927.

[29] Meng, X., Zhang, J., Sun, Z., Zhou, L., Deng, G., Li, S., Li, W., Gong, P., and Cai, L. (2018) Hypoxia-triggered single molecule probe for high-contrast NIR II/PA tumor imaging and robust photothermal therapy, Theranostics 8, 60256034.

[30] Zhang, C., Gao, R., Zhang, L., Liu, C., Yang, Z., and Zhao, S. (2020) Design and Synthesis of a Ratiometric Photoacoustic Probe for In Situ Imaging of Zinc lons in Deep Tissue In Vivo, Analytical Chemistry 92, 6382-6390.

[31] Wu, Y., Huang, S., Wang, J., Sun, L., Zeng, F., and Wu, S. (2018) Activatable probes for diagnosing and positioning liver injury and metastatic tumors by multispectral optoacoustic tomography, Nature Communications 9 , 3983.

[32] Sun, L., Wu, Y., Chen, J., Zhong, J., Zeng, F., and Wu, S. (2019) A Turn-On Optoacoustic Probe for Imaging Metformin-Induced Upregulation of Hepatic Hydrogen Sulfide and Subsequent Liver Injury, Theranostics 9, 7789.

[33] Ma, T., Zheng, J., Zhang, T., and Xing, D. (2018) Ratiometric photoacoustic nanoprobes for monitoring and imaging of hydrogen sulfide in vivo, Nanoscale 10, 13462-13470.

[34] Cheng, P., Chen, W., Li, S., He, S., Miao, Q., and Pu, K. (2020) Fluoro-Photoacoustic Polymeric Renal Reporter for RealTime Dual Imaging of Acute Kidney Injury, Advanced Materials 32, 1908530.

[35] Gao, W., Li, X., Liu, Z., Fu, W., Sun, Y., Cao, W., Tong, L., and Tang, B. (2019) A Redox-Responsive Self-Assembled Nanoprobe for Photoacoustic Inflammation Imaging to Assess Atherosclerotic Plaque Vulnerability, Analytical Chemistry 91, 1150-1156.

[36] Zeng, Q., Wu, Y., and Zhang, T. (2019) Photoacoustic nanoprobe for $\beta$-galactosidase activity detection and imaging in vivo, Journal of Innovative Optical Health Sciences 12, 1941001.

[37] Gao, X., Ma, G., Jiang, C., Zeng, L., Jiang, S., Huang, P., and Lin, J. (2019) In Vivo Near-Infrared Fluorescence and Photoacoustic Dual-Modal Imaging of Endogenous 
Alkaline Phosphatase, Analytical Chemistry 91, 71127117.

[38] Huang, J., Wu, Y., Zeng, F., and Wu, S. (2019) An Activatable Near-Infrared Chromophore for Multispectral Optoacoustic Imaging of Tumor Hypoxia and for Tumor Inhibition, Theranostics 9, 7313-7324.

[39] Lucero, M. Y., and Chan, J. (2020) Towards Personalized Medicine: Photoacoustic Imaging Enables Companion Diagnosis and Targeted Treatment of Lung Cancer.

[40] Yuan, L., Lin, W., Zhao, S., Gao, W., Chen, B., He, L., and Zhu, S. (2012) A Unique Approach to Development of NearInfrared Fluorescent Sensors for in Vivo Imaging, Journal of the American Chemical Society 134, 13510-13523.

[41] Debacq-Chainiaux, F., Erusalimsky, J. D., Campisi, J., and Toussaint, O. (2009) Protocols to detect senescenceassociated beta-galactosidase (SA-ßgal) activity, a biomarker of senescent cells in culture and in vivo, Nature Protocols 4, 1798-1806.

[42] Asanuma, D., Sakabe, M., Kamiya, M., Yamamoto, K., Hiratake, J., Ogawa, M., Kosaka, N., Choyke, P. L., Nagano, T., Kobayashi, H., and Urano, Y. (2015) Sensitive $\beta$ galactosidase-targeting fluorescence probe for visualizing small peritoneal metastatic tumours in vivo, Nature Communications 6, 6463.

[43] Wong, R. H. F., Kwong, T., Yau, K.-H., and Au-Yeung, H. Y. (2015) Real time detection of live microbes using a highly sensitive bioluminescent nitroreductase probe, Chemical Communications 51, 4440-4442.

[44] Qin, W., Xu, C., Zhao, Y., Yu, C., Shen, S., Lin, L., and Huang, W. (2018) Recent progress in small molecule fluorescent probes for nitroreductase, Chinese Chemical Letters 29 , 1451-1455.

[45] Iwashita, H., Castillo, E., Messina, M. S., Swanson, R. A., and Chang, C. J. (2021) A tandem activity-based sensing and labeling strategy enables imaging of transcellular hydrogen peroxide signaling, Proceedings of the National Academy of Sciences 118, e2018513118.

[46] Montoya, L. A., and Pluth, M. D. (2012) Selective turn-on fluorescent probes for imaging hydrogen sulfide in living cells, Chemical Communications 48, 4767-4769.
[47] Saldova, R., Royle, L., Radcliffe, C. M., Abd Hamid, U. M., Evans, R., Arnold, J. N., Banks, R. E., Hutson, R., Harvey, D. J., Antrobus, R., Petrescu, S. M., Dwek, R. A., and Rudd, P. M. (2007) Ovarian Cancer is Associated with Changes in Glycosylation in Both Acute-Phase Proteins and IgG, Glycobiology 17, 1344-1356.

[48] Guo, W., Hiratake, J., Ogawa, K., Yamamoto, M., Ma, S. J., and Sakata, K. (2001) Beta-D-glycosylamidines: potent, selective, and easily accessible 1-glycosidase inhibitors, Bioorg Med Chem Lett 11, 467-470.

[49] Watts, M. E., Pocock, R., and Claudianos, C. (2018) Brain Energy and Oxygen Metabolism: Emerging Role in Normal Function and Disease, Frontiers in Molecular Neuroscience 11

[50] Huang, W.-J., Zhang, X., and Chen, W.-W. (2016) Role of oxidative stress in Alzheimer's disease, Biomed Rep 4, 519-522.

[51] Yang, J., Yang, J., Liang, S. H., Xu, Y., Moore, A., and Ran, C. (2016) Imaging hydrogen peroxide in Alzheimer's disease via cascade signal amplification, Scientific Reports 6 , 35613.

[52] Yang, J., Zhang, X., Yuan, P., Yang, J., Xu, Y., Grutzendler, J., Shao, Y., Moore, A., and Ran, C. (2017) Oxalatecurcumin-based probe for micro- and macroimaging of reactive oxygen species in Alzheimer's disease, Proceedings of the National Academy of Sciences 114, 12384.

[53] Dinkins, M. B., Dasgupta, S., Wang, G., Zhu, G., He, Q., Kong, J. N., and Bieberich, E. (2015) The 5XFAD Mouse Model of Alzheimer's Disease Exhibits an Age-Dependent Increase in Anti-Ceramide IgG and Exogenous Administration of Ceramide Further Increases AntiCeramide Titers and Amyloid Plaque Burden, $J$ Alzheimers Dis 46, 55-61.

[54] Cai, S., Liu, C., Jiao, X., Zhao, L., and Zeng, X. (2020) A lysosome-targeted near-infrared fluorescent probe for imaging endogenous cysteine (Cys) in living cells, Journal of Materials Chemistry B 8, 2269-2274. 
Entry for the Table of Contents

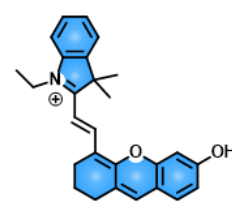

Repurposed for PA

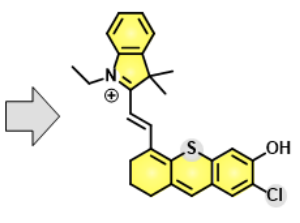

Optimized for PA

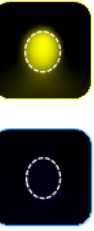

General platform for

probe development

4.8-fold increase in

sensitivity

Ratiometric imaging

enabled

We have developed PA-HD, a new sulfur-substituted dye platform exhibiting enhanced properties for photoacoustic probe development. To demonstrate generalizability, we employed PA-HD to access probes for beta-galactosidase and nitroreductase activities, as well as $\mathrm{H}_{2} \mathrm{O}_{2}$. Each probe was tested in live cells and in vivo. For instance, the $\mathrm{H}_{2} \mathrm{O}_{2}$ probe was employed to visualized oxidative stress in an Alzheimer's disease model.

Institute and/or researcher Twitter usernames: @ChanLabUIUC @MiricaGroup 